\title{
Evaluation of Prognostic Predictors of Mangled Extremity Severity Scoring System on the Outcome of Traumatic Extremities Injuries at Emergency Department in Suez Canal University Hospitals
}

\author{
Ahmed A. Essa*, Islam M. El-Shaboury, Yasmin E. El-Beltagy \\ Department of Emergency Medicine, Faculty of Medicine, Suez Canal University, Egypt
}

\begin{abstract}
Background: Severe traumatic lower limbs injuries have been associated with high incidence of multiple systems involved (integument, nerve, bone, and vascular structures).That's make difficulty and stress on surgeon's decision making either to amputate or preserve the injured limbs. The Mangled Extremity Severity Score (MESS) was developed in Seattle (Johansen et al 1990), based on both retrospective and prospective analysis of admission data of patients with severe limb injuries. Aim: evaluation of the prognostic predicting factors of outcome of traumatic extremities patients using Mangled Severity Scoring System at Emergency Department in Suez Canal University Hospital in order to help surgeon to make decision either to preserve or to amputate. Patients and Methods: This is a descriptive study (cross sectional), conducted on 60 patients with severe extremities injuries that met the criteria of the Mangled Extremity Severity Scoring attending to the Emergency Department (ED) at Suez Canal University Hospital. Results: This study showed that $83.33 \%$ of the patients had associated fractures, $8.33 \%$ of them had pneumothorax, $5 \%$ of them had abdominal collection and $3.33 \%$ of them had brain injuries. This study showed that $75 \%$ of the studied patients had MESS less than 7 while $25 \%$ of them had MESS > or $=7$. The Mangled scoring system was a good predictor of amputation among the studied patients with sensitivity of $71.4 \%$, specificity of $100 \%$ and $90 \%$ accuracy. Conclusion: Most of the patients had Mangled Extremity Severity Score less than 7. The MESS showed statistically significant difference between the amputated and the nonamputated patients with $90 \%$ accuracy in prediction of amputation.
\end{abstract}

Key words: MESS, ED

\section{Introduction}

Severe traumatic lower limbs injuries have been associated with high incidence of multiple systems involved (integument, nerve, bone, and vascular structures $)^{(1)}$. That's make difficulty and stress on surgeon's decision making either to amputate or preserve the injured limbs ${ }^{(1)}$. Attempts to qualify the

*Corresponding Author: abdelhady200@yahoo.com 
severity of the trauma and to establish numerical guidelines to whether amputate or salvage the limb have been proposed by several authors. The Mangled Extremity Severity Score (MESS) was developed in Seattle, based on both retrospective and prospective analysis of admission data of patients with severe limb injuries. Four variables determine skeletal and soft-tissue injury, limb ischemia, surgical shock, and the age of the patient ${ }^{(2,3)}$. The aim of the work is to evaluate the prognostic predictors of Mangled Extremity Severity Scoring system on the outcome of traumatic extremities patients attending emergency department in Suez Canal university hospitals.

\section{Patients and Methods}

This is a descriptive cross sectional study that was conducted on 60 patients attending to the Emergency Department (ED) at Suez Canal University Hospital with severe extremities injuries. All patients met the criteria of the Mangled Extremity Severity Scoring. Data was collected in pre-organized data sheet by the researcher from patients fulfilling inclusion and exclusion criteria. All patients were clinically assessed and managed using the $A B C D E$ protocol. Also, by using the Mangled Extremity Severity Score (MESS) [Skeletal/soft tissue injury-Limb ischemia- Shock- Age], to evaluate the prognostic predicting factors of the outcome of traumatic extremities patients. The patients were followed up and recorded till one of the following outcomes is reached: i) Amputated, ii) Had surgical reconstruc- tion, iii) I.C.U admission, iv) Died at the emergency room.

\section{Results}

This descriptive study was conducted to evaluate the prognostic predictors of outcome of traumatic extremities injuries using Mangled Extremity Severity Score (MESS) depending on four variables determining skeletal and soft-tissue injury, limb ischemia, surgical shock, and the age of the patient. This study was conducted on 60 Polytraumatized patients with severe extremities injuries met the criteria of the Mangled Extremity Severity Scoring attending to the Emergency Department (ED) at Suez Canal University Hospital. In this study, the mean age was $30.8 \pm 12.2$ year, $38.3 \%$ of them were in the age group between 25-34 years and $91.6 \%$ of the patients were males. Lower limb injuries (only) represented $71.67 \%$ of all injuries, upper limb injuries (only) as they represented $25 \%$ of all injuries, while injuries in both limbs represented $3.33 \%$. The right side (only) was the most common side to be affected about $50 \%$ of all injuries. This study showed that the leg was the most common site to be injured in the lower limb (73.3\%), while, the hand was the most site to be injured in the upper limb (35.2\%). Regarding the mechanism of injury, road traffic accidents were responsible for $83.3 \%$ of injuries followed by industrial injuries which were responsible for $16.67 \%$. This study showed that $86.67 \%$ the patients had active bleeding with the venous injury represented $67.31 \%$ of the vascular injur- 
Table 1: Comparison between patients with and without amputation regarding age, initial clinical evaluation and mechanism of trauma

\begin{tabular}{|l|ccc|}
\hline & \multicolumn{2}{|c}{ Amputation } & p- \\
& No & Yes & value \\
\hline Age (Yrs) & $29.28 \pm 12.47$ & $33.71 \pm 11.45$ & 0.2 \\
SBP & $110.77 \pm 8.7$ & $102.38 \pm 9.95$ & 0.003 \\
DBP & $71.54 \pm 7.45$ & $62.38 \pm 8.89$ & 0.003 \\
HR & $90.77 \pm 7.03$ & $94.76 \pm 17.42$ & 0.3 \\
Trauma Mechanism & & & \\
\multicolumn{1}{|c|}{ - Motor car accident } & $31(79.49 \%)$ & $19(90.48 \%)$ & 0.3 \\
$\quad$ - Industrial injuries & $8(20.51 \%)$ & $2(9.52 \%)$ & \\
\hline
\end{tabular}

$\xi=$ data are presented as mean \pm SD; *Statistical significance at $\mathrm{p} \leq 0.05 ; \mathrm{SBP}=$ Systolic Blood Pressure; DBP= Diastolic Blood Pressure, HR= Heart Rate

ies while the arterial injury represented $32.69 \%$ of all vascular injuries. Half $(50 \%)$ of the patients had absent distal pulsation in the affected limb. Regarding the sensory function, $96.67 \%$ of the patients had hypothesia. Regarding the motor function, the entire patients had muscle weakness in the affected limb, $83.3 \%$ of the studied patients had associated fractures, $8.33 \%$ had pneumothorax injuries, $5 \%$ had abdominal collection and $3.33 \%$ had associated brain injuries. This study showed that Mangled score had statistically significant difference between the amputated and the nonamputated patients as of the 21 patients who had amputations, 15 (71.4\%) had Mangled score equal or more than 7 while $6(28.6 \%)$ of them had Mangled score $<7$ and 39 patients avoided amputation and all of them had Mangled score $<7$ with good correlation between the Mangled score in relation to the actual performed amputation. Mangled score had sensitivity of $71.4 \%$, specificity of $100 \%$ and $90 \%$ accuracy in prediction of amputation. This study revealed that 21 of the studied patients had amputation (35\%) while 39 of them avoided amputation and had surgical reconstruction and repair (65\%) (Figure 1). Mangled score had the ability to predict amputation in relation to the actual performed amputation among the studied patients (Figure 1). Regarding the initial clinical evaluation of the patients, the mean of SBP in the amputated group and the not amputated group of the studied patients was $102.38 \pm 9.95$ and $110.77 \pm 8.7 \mathrm{mmHg}$ respectively and the mean of DBP in the amputated group and the nonamputated group of the studied patients was $62.38 \pm 8.89$ and $71.54 \pm 7.45$ $\mathrm{mmHg}$ respectively which showed statistically significant difference between both groups regarding the decision of amputation. This concludes that SBP and DBP were the vital signs that showed statistically significant difference between the amputated and 
non-amputated patients (Table 1). The mean of the age of the amputated group and the non- amputated group was $33.71 \pm 11.45$ and 29.28 \pm 12.47 , mechanism of trauma and HR had no statistically significant difference between both groups regarding the decision of amputation (Table 1). Regarding the initial clinical evaluation of the affected limb, the type of bleeding and presence or absence of distal pulsation showed statistically significant difference between the amputated and non-amputated patients with p-value of 0.01 and 0.001 respectively (Table 2). The mean of the HB level between the patients with and without amputation was $10.2 \pm 1.2$ and $10.6 \pm 1.3 \mathrm{~g} \%$ respectively which had no statistically significant difference.
Moreover, complete blood count (CBC) parameters were not different between the amputated and non-amputated patients (Table 3). Regarding the clinical evaluation of the site of injury, this study found that the absence of distal pulsation in the injured limb and the arterial bleeding had statistically significant difference between patients with and without amputation. Amputation was done among 21 patients among whom 15 patients (71.4\%) has Mangled score with preference of amputation. All patients who had no amputation has Mangled score with preference of not to amputate. Mangled score had statistically significant difference regarding the prediction of the limb amputation or salvation among the studied patients (Table 4).

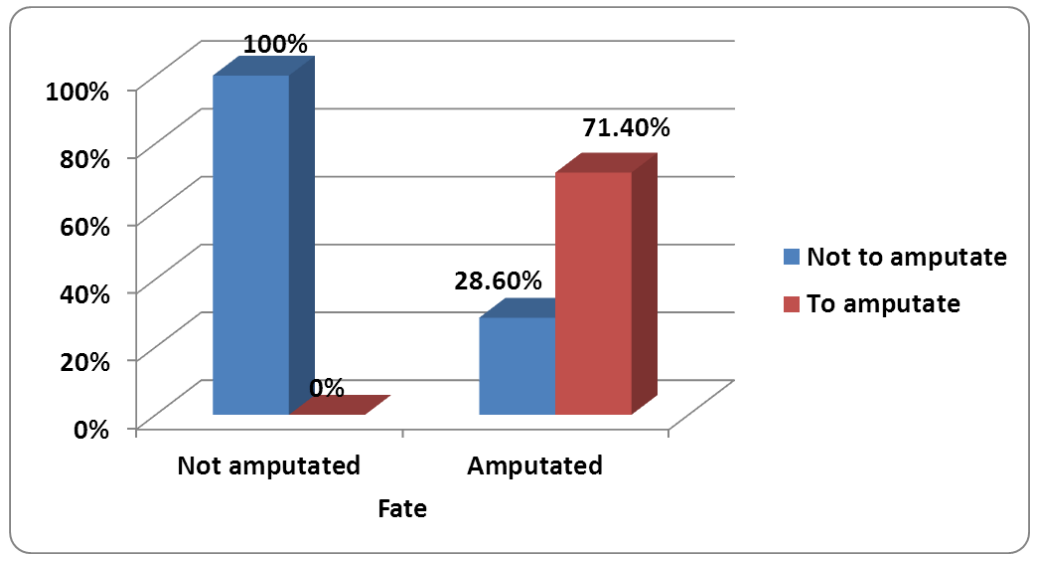

Figure 1: Decision of amputation according to Mangled score in relation to actual performed amputation

\section{Discussion}

Trauma is a serious global health problem, accounting for about one in 10 deaths worldwide ${ }^{(4,5)}$, and five million deaths per year, of which 1 million are in Europe $^{(6)}$. Severe traumatic lower limbs injuries have been associated with high incidence of multiple systems involved (integument, nerve, bone, and vascular 
structures $^{(4)}$. Several limb salvage scoring systems have been devised to aid clinicians to take their decision of when to salvage or to amputate. The developers of these scoring systems attempted to validate them by demonstrating high rates of specificity and sensitivity in predicting limb salvage ${ }^{(6,7)}$.

Table 2: Comparison between patients with and without amputation regarding clinical evaluation of site of injury

\begin{tabular}{|l|ccc|}
\hline & Amputation & P-value \\
\hline Active bleeding & No & $3(14.29 \%)$ & 0.9 \\
$\quad$ No & $5(12.82 \%)$ & $18(85.71 \%)$ & \\
$\quad$ Yes & $34(87.18 \%)$ & $8(44.44 \%)$ & $0.01^{*}$ \\
Type of bleeding & $27(79.41 \%)$ & $10(55.56 \%)$ & \\
$\quad$ Venous & $7(20.59 \%)$ & 0 & $0.001^{*}$ \\
$\quad$ Arterial & $30(76.92 \%)$ & $21(100 \%)$ & \\
$\begin{array}{l}\text { Distal pulse } \\
\quad \text { Intact }\end{array}$ & $9(23.08 \%)$ & $2(9.52 \%)$ & $0.05^{*}$ \\
$\quad$ No & 0 & $19(90.48 \%)$ & \\
$\begin{array}{l}\text { Sensory function } \\
\text { Parethesia }\end{array}$ & $39(100 \%)$ & 0 & 0.3 \\
$\quad$ Hypothesia & $2(5.13 \%)$ & $21(100 \%)$ & \\
Distal neurovascular & $37(94.87 \%)$ & & \\
$\quad$ Intact & & & \\
$\quad$ Injury & & & \\
\hline
\end{tabular}

*Statistical significance at $p \leq 0.05$

The Mangled Extremity Severity Score (MESS) was developed in Seattle ${ }^{(8)}$, based on both retrospective and prospective analysis of admission data of patients with severe limb injuries. Four variables are used in the score which are: skeletal and soft-tissue injury, limb ischemia, shock, and the age of the patients ${ }^{(2,3)}$. This is a descriptive study conducted in the Emergency Department of the Suez Canal University Hospital to evaluate the prognostic predictors of Mangled Extremity Severity Scoring System on the Outcome of Traumatic Extremities Patients. Almost $50 \%$ of injury-related mortality is in young people between the ages of 15 and 44 years $^{(6)}$. In this study, the mean age was $30.83 \pm 12.21$ year, $38.33 \%$ of them were in the age group between 25-34 years and $91.6 \%$ of the patients were males. These results match the results of another study conducted by Kumar et al, in which the mean age of the patients was 34.5 years and most of them were males $84.6 \%^{(4)}$. Also these results match the results of another study performed by David $S$ et al, in which the mean age of the patients was 30.6 years and males accounted for $85.4 \%$ of the patients ${ }^{(5)}$. Another study by Karami et al, matches the results of 
this study in which the mean age of the patients was 28 years and $90 \%$ of the patients were males ${ }^{(6)}$. In this study, lower limb injuries(only) represented $71.67 \%$ of all injuries and they were more frequent than upper limb injuries (only) as they represented $25 \%$ of all injuries while $3.33 \%$ due to injuries in both limbs. These results match the results of another study performed in which of the severely damaged limbs, 30\% upper and $70 \%$ lower extremities ${ }^{(6)}$. This study showed that the right side (only) was the most common side to be affected about $50 \%$ of all injuries as these results match the results of a previous study, in which the right side was commonly injured about $57 \%{ }^{(4)}$. This study showed that the leg was the most common site to be injured in the lower limb $73.33 \%$ and this matches the results of a study performed by Kumar et al, in which crush injury of leg was observed in $80 \%$ of injured limbs ${ }^{(4)}$. Also this study showed that the hand was the most site to be injured in the upper limb $35.29 \%$.

Table 3: Comparison between patients with and without amputation regarding laboratory evaluation

\begin{tabular}{|c|c|c|c|}
\hline & \multicolumn{2}{|c|}{ Amputation } & \multirow{2}{*}{$\mathrm{p}$-value } \\
\hline & No & Yes & \\
\hline Hemoglobin (gm/dl) & $10.6 \pm 1.3$ & $10.2 \pm 1.2$ & 0.2 \\
\hline $\operatorname{TLC}\left(\times 10^{3}\right)$ & $11.2 \pm 1.58$ & $11.1 \pm 1.5$ & 0.8 \\
\hline Platelet $\left(\times 10^{3}\right)$ & $255.1 \pm 55.9$ & $230 \pm 36.3$ & 0.05 \\
\hline
\end{tabular}

Data are presented as mean \pm SD; *Statistical significance at $p \leq 0.05$

Table 4: Decision of amputation according to Mangled score in relation to actual performed amputation

\begin{tabular}{|c|c|c|c|}
\hline \multirow{2}{*}{ Decision of Mangled score } & \multicolumn{2}{|c|}{ Performed amputate } & \multirow{2}{*}{ p-value } \\
\hline & No & Yes & \\
\hline $\begin{array}{l}\text { Not to amputate }(<7) \\
\text { To amputate }(>\text { or }=7)\end{array}$ & $\begin{array}{c}39(100 \%) \\
0\end{array}$ & $\begin{array}{l}6(28.6 \%) \\
15(71.4 \%)\end{array}$ & $0.001 *$ \\
\hline
\end{tabular}

*Statistical significance at $p \leq 0.05$

Regarding the mechanism of injury, this study showed that road traffic accidents were responsible for $83.33 \%$ of injuries followed by industrial injuries which were responsible for $16.67 \%$. These results agree with the results of another study performed by Al-Salman $\mathrm{M}$ et al, in which the majority of vascular injuries were caused by blunt trauma and $91 \%$ of those were caused by road traffic collisions $^{(9)}$. This study showed that $86.67 \%$ the patients had active bleeding with the venous injury represented $67.31 \%$ of the vascular injuries while the 
arterial injury represented $32.69 \%$ of all vascular injuries which does not match with the results performed by Kauvar et al, in which venous injury was present in $26.6 \%$ of injuries while arterial injury was $73.4 \% .{ }^{(5)}$ This study showed that $50 \%$ of the patients had absent distal pulsation in the affected limb which matches the results of study, in which pulse deficit was documented in $50.7 \%$ of the patients on arrival to the emergency room ${ }^{(10)}$. These results do not match the results of another study conducted by Bosse et al, in which absence of distal pulsation presented in $24.6 \%$ of the patients and this may be due to the large sample size $(n=545)$ while the present study had only 60 patients in the sample size ${ }^{(11)}$. Regarding the sensory function, this study showed that $96.67 \%$ of the patients had hypothesia which doesn't match the results of another study performed by Bosse et al, in which $20 \%$ of the patients had sensory deficits on presenting to the emergency room and this may be due the large sample size $(n=545)$ while the present study had only 60 patients in the sample size ${ }^{(11)}$. Regarding the motor function, this study found that the entire patient had muscle weakness in the affected limb. These results match the results of another study, in which $81.5 \%$ of the patients ${ }^{(10)}$. This study showed that $83.33 \%$ of the studied patients had associated fractures, $8.33 \%$ of them had pneumothorax injuries, $5 \%$ of them had abdominal collection and $3.33 \%$ had associated brain injuries. These results match the results of Karami et al, in which all patients had fractures ${ }^{(6)}$. These results match also the results of another study, in which $3.17 \%$ of the patient had concomitant chest injuries ${ }^{(12)}$. This study showed that Mangled score had a statistically significant difference between the amputated and the nonamputated patients; of the 21 patients who had amputations, 15 (71.4\%) had Mangled score equal or more than 7 while 6 (28.6\%) of them had Mangled score $<7$ and it was found that 39 patients avoided amputation and all of them had Mangled score $<7$ with good correlation between the Mangled score in relation to the actual performed amputation. These results match the results of another study performed by Feritas et al, in which 8 patients exposed to amputation, 6 of them (75\%) had Mangled score equal or more than 7 while only 2 (25\%) had Mangled score less than 7 and it was found that 12 patients avoided amputation and all of them had Mangled score less than $7^{(13)}$. This study showed that Mangled score had sensitivity of $71.4 \%$, specificity of $100 \%$ and $90 \%$ accuracy in prediction of amputation. These results match the results of another study, in which the Mangled score had a high specificity (98\%) and a high sensitivity $(91 \%)^{(4)}$, also these results match the results of another study conducted by Karami et al, in which the Mangled score demonstrated a high specificity $80 \%$ and high sensitivity $87 \%$ for all of injured limb analysis $^{(6)}$. This study revealed that 21 of the studied patients had amputation (35\%) while 39 of them avoided amputation and had surgical reconstruction and repair $(65 \%)$. These results 
match the results of another study, in which $40 \%$ of the patients had amputation while $60 \%$ of them had limb salvation ${ }^{(13)}$, also they match the results of Karami et al, in which $25 \%$ of the patients had amputation and $75 \%$ of them had avoided the amputation ${ }^{(6)}$ Regarding the initial clinical evaluation of the patients, this study found that the mean of SBP in the amputated group and the non-amputated group of the studied patients was $102.3 \pm 9.9$ and $110.7 \pm 8.7 \mathrm{mmHg}$ respectively and the mean of DBP in the amputated group and the non-amputated group of the studied patients was $62.3 \pm 8.8$ and $71.5 \pm 7.4 \mathrm{mmHg}$ respectively which showed statistically significant difference between both groups regarding the decision of amputation. In contrast, Feritas et al, reported that the mean of SBP in the amputated group and the salvaged group was 107 and $130 \mathrm{mmHg}$ respectively which showed no statistically significant difference ${ }^{(13)}$. This study showed that the mean of the age of the amputated group and the nonamputated group was $33.71 \pm 11.45$ and $29.28 \pm 12.47$, mechanism of trauma and HR had no statistically significant difference between both groups regarding the decision of amputation. These results match the results of a study performed by Feritas et al, in which the mean age of the amputated group and the non-amputated group was 40.5 and 30 respectively which showed no statistically significant difference ${ }^{(13)}$ Regarding the clinical evaluation of the site of injury, this study found that absence of distal pulsation in the injured limb and the arterial bleeding had statistically significant difference between patients with and without amputation. These results match the results of Bosse et al, in which absence of distal pulsation at time of examination and associated arterial injuries were more indicative of poor outcome and amputation $^{(11)}$. This study found that the mean of the HB level between the patients with and without amputation was $10.2 \pm 1.2$ and $10.6 \pm 1.3 \mathrm{~g} \%$ respectively which had no statistically significant difference.

\section{Conclusion}

The MESS had statistically significant difference between the amputated and the non-amputated patients with 90\% accuracy in prediction of amputation. The MESS had sensitivity of $71.4 \%$, specificity of $100 \%$ among the studied patients.SBP and DBP were the only vital signs that showed statistically significant difference between the amputated and non-amputated patients regarding decision of amputation. Absence of distal pulsation of the injured extremities and the type of bleeding had statistically significant difference between the amputated and non-amputated patients regarding decision of amputation. Also it was found that age, $H R$, mechanism of injury, the presence of active bleeding and the presence of sensory/motor dysfunction had no statistically significant difference between the amputated and not amputated patients regarding decision of amputation. 


\section{References}

1. Fodor L, Sobec R, Sita-Alb L, Fodor $M$, Ciuce $C$. Mangled lower extremity: can we trust the amputation scores? .Int J Burn Trauma 2012;2(1):51-58.

2. Helfet DL, Howey $T$, Sanders R, Johansen K. Limb salvage versus amputation. Preliminary results of the Mangled Extremity Severity Score . Clin Orthop RelatedRes. 1990;(256):80-6.

3. Robertson PA, British Editorial Society of Bone and Joint Surgery J Bone joint Surg. [Br] 1991 ; 73-B:8l68.

4. Kumar MK, Badole CM, Patond KR. Salvage versus amputation: Utility of mangled extremity severity score in severely injured lower limbs. Indian J Orthop 2007; 41(3): 183-187.

5. Kauvar DS, Sarfati MR, Kraiss LW. National trauma databank analysis of mortality and limb loss in isolated lower extremity vascular trauma. J Vasc Surg 2011;53: 1598-603.

6. Karami M, Sadat MM, Tavakkoli H, Taghavi M, Golbakhsh MR. Predictive validity testing of severely injured limb scoring systems. Arch Iranian Med 2004;7(1): 31-36.

7. Durham RM, Mistry BM, Mazuski JE, Shapiro M, Jacobs D. Outcome and utility of scoring systems in the management of the mangled extremity. Am J Surg. 1996; 172: 569 -74 .

8. Johansen $\mathrm{K}$, Daines $\mathrm{M}$, Howey $\mathrm{T}$, Helfet D, Hansen ST Jr. Objective criteria accurately predict amputation following lower extremity trauma. J Trauma. 1990; 30(5):568-72
9. Al-Salman $\mathrm{M}$, Al-Khawashki $\mathrm{H}$, Sindigki $A$ et al. Vascular injuries associated with limb fractures. Injury 1997;28: 103-107.

10. Russell WL, Sailors DM, Whittle TB et al. Limb Salvage Versus Traumatic Amputation. Ann. Surg 1991;213(5): $473-480$.

11. Bosse MJ, Mackenzie EJ, Kellam JF et al. An Analysis of Outcomes of Reconstruction or Amputation of Leg-Threatening Injuries. N Engl J Med 2002;347: 1924-31.

12. Korompilias AV, Beris $\mathrm{AE}, \mathrm{MG}$ Lykissas et al. The mangled extremity and attempt for limb salvage. Journal of Orthopaedic Surgery and Research 2009;4(4): 1-6.

13. Feritas DD, Oliveira CD, Rasslan S et al. Preliminary Results of a Prospective Study on Severe Lower Limb Trauma: Analysis of Laboratory Tests as Predictors of Amputation. Panam J Trauma Critical Care Emerg Surg 2013;2(1): 58-61. 
